\title{
Free vibration of rectangular plates with attached discrete sprung masses
}

\author{
Ding Zhou ${ }^{\mathrm{a}, *}$ and Tianjian $\mathrm{Ji}^{\mathrm{b}}$ \\ ${ }^{a}$ College of Civil Engineering, Nanjing University of Technology, Nanjing, People's Republic of China \\ ${ }^{\mathrm{b}}$ School of Mechanical, Aerospace and Civil Engineering, The University of Manchester, Manchester, UK
}

Received 11 September 2008

Revised 18 September 2010

\begin{abstract}
A direct approach is used to derive the exact solution for the free vibration of thin rectangular plates with discrete sprung masses attached. The plate is simply supported along two opposite edges and elastically supported along the two other edges. The elastic support can represent a range of boundary conditions from free to clamped supports. Considering only the compatibility of the internal forces between the plate and the sprung masses, the equations of the coupled vibration of the plate-spring-mass system are derived. The exact expressions for mode and frequency equations of the coupled vibration of the plate and sprung masses are determined. The solutions converge steadily and monotonically to exact values. The correctness and accuracy of the solutions are demonstrated through comparison with published results. A parametric study is undertaken focusing on the plate with one or two sprung masses. The results can be used as a benchmark for further investigation.

The solution provided in the paper is general and includes several special cases, such as the plate with classical boundary conditions, the plate attached with discrete rigid masses, the plate supported by discrete springs and the plate restricted by rigid vertical point-supports.
\end{abstract}

Keywords: Coupled vibration, eigenfrequency, plate, sprung mass, exact solution

\section{Introduction}

There are several situations where the vibration of plates with attached discrete masses needs to be considered, one example being a structure supporting people [1]. In this situation the person (or crowd) can be represented by mass-spring-damper model(s). Another example occurs when the vibrations from a machine are reduced using a rubber membrane between it and the floor, thereby creating a sprung-mass system. This paper presents a theoretical study which provides an improved understanding of the vibration of couple spring-mass and plate systems.

There are three possible ways to study the above problem. First by converting the floor into a single degreedegree-of-freedom (SDOF) system and the discrete sprung masses into a single sprung mass; thus forms a two degree-of-freedom system. The advantage of the method is that it greatly simplifies the solution of the problem. On the other hand, the model neglects higher modes of vibration of the system. Second using a FE package makes direct modelling. This method is simple and quick for particular solutions, but it may be tedious for parametric studies of the plate-spring-mass problem considering several variable parameters. Thirdly using analytical studies to derive expressions for the solution of the problem in order to conduct parametric studies thereby provides an improved understanding of the problem. The paper investigates the problem using the third route.

The general study to vibration characteristics of rectangular plates with arbitrarily located point-supports came from Fan and Cheung [2] using the spline finite strip. Kim and Dickinson [3] used the Lagrangian multiplier method

\footnotetext{
${ }^{*}$ Corresponding author: Dr. Ding Zhou, Professor, College of Civil Engineering, Nanjing University of Technology, Nanjing 210009, People's Republic of China. Tel.: +86 25 84316695; E-mail: dingzhou57@yahoo.com.
} 


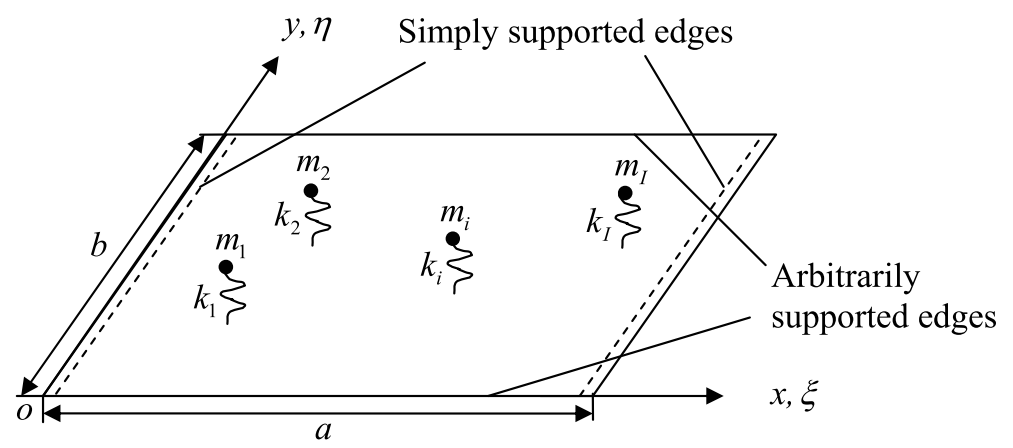

Fig. 1. A rectangular plate having two opposite edges simply supported and other two opposite edges arbitrarily supported, with discrete sprung masses attached.

combining with the orthogonally generated polynomials to study the rectangular plates with point-supports. Zhou [4] and Cheung and Zhou [5] used the static beam functions as admissible functions to study tapered rectangular plates with point-supports and composite rectangular plates with point-supports respectively. Moreover, the 3-D vibration of composite plates with internal point-supports has been studied by Zhou et al. [6] using the finite layer method. Bergman et al. [7] derived the dynamic Green function for rectangular plates having two opposite edges simply supported and attached by substructures. Li [8] gave the exact solution of rectangular plates with two opposite edges simply supported and with line-concentrated masses or elastic line-supports. Avalos et al. $[9,10]$ used the optimized Rayleigh-Ritz approach and sinusoidal eigenfunction expansion method respectively to study the free vibration of a circular plate carrying an elastically attached mass at its centre and a simply supported rectangular plate having an elastically attached mass at an arbitrary position. Wong [11] applied the Rayleigh-Ritz method to study the effect of distributed mass on plate vibration behaviour. Wu et al. [12] used the mode expansion method to investigate the effect of various concentrated elements. Jacquot [13] used the two-dimensional Fourier series to study the random vibration of rectangular plate attached with absorbers. Chiba and Sugimoto [14] studied the vibration characteristics of a cantilevered rectangular plate attached by a sprung mass in detail. Liew et al. [15] studied the free vibration of arbitrarily shaped Mindlin plates with internal point-supports. McMillan and Keane [16, 17] investigated the possibility of using attached masses to control the vibration of rectangular plates. Adhikari et al. [18,19] experimentally studied the effect of uncertainty quantification on structural dynamics. Although some investigators $[10,12,13]$ used the two-dimensional Fourier sinusoidal series to obtain the exact solutions of the dynamic characteristics of plates attached with elastic/rigid masses, such a method is only applicable to plates with four edges simply supported. It should be mentioned that the existing studies mainly focused on plates with classical boundary conditions and with one sprung mass. No study on plates with elastic boundary conditions and with multi-spring- masses has been found.

In the present study, the exact analytical solution for a thin rectangular plate attached to sprung masses is derived from the governing differential equations. The plate is simply supported along two opposite edges and elastically supported along the other edges. The eigenvalue equations are obtained by considering the compatibility of internal forces between the plate and the sprung masses at the attached positions. The order of the eigenvalue equations is equal to the number of the attached sprung masses. The eigenfrequencies are obtained using the root searching method. Several special cases can be derived from the given solutions. A parametric study is conducted to examine the effects of the relative mass and stiffness, and the locations of the sprung mass on the dynamic characteristics of the coupled system.

\section{Governing differential equations}

Consider a uniform thin rectangular plate with a length of $a$ and a width of $b$ attached to $I$ sprung masses as shown in Fig. 1. It is assumed that the plate is simply supported along two opposite edges $x=0$ and $x=a$. According to the theory of thin plate vibration when damping is neglected, the governing differential equation of the plate under the forces from the sprung masses is 


$$
D\left(\frac{\partial^{4} w}{\partial x^{4}}+2 \frac{\partial^{4} w}{\partial x^{2} \partial y^{2}}+\frac{\partial^{4} w}{\partial x^{4}}\right)+\rho h \frac{\partial^{2} w}{\partial t^{2}}=f(x, y, t)
$$

where $w$ is the transverse deflection of the plate, $\rho$ is the mass density per unit volume of the plate, $h$ and $D$ are the thickness and the flexural rigidity of the plate, $f(x, y, t)$ is the action forces of the sprung masses on the plate. The simply supported boundary conditions of the plate are

$$
w=0, \quad \frac{\partial^{2} w}{\partial x^{2}}+\nu \frac{\partial^{2} w}{\partial y^{2}}=0 \text { at } x=0 \text { and } a
$$

In which, $\nu$ is the Poisson's ratio. The loading term $f(x, y, t)$ can be expressed as:

$$
f(x, y, t)=\sum_{i=1}^{I} p_{i}(t) \delta\left(x-x_{i}\right) \delta\left(y-y_{i}\right)
$$

where $p_{i}(t)$ is the force of the $i$ th sprung mass on the plate, $x_{i}$ and $y_{i}$ are the coordinates of the $i$ th sprung mass, $\delta\left(x-x_{i}\right)$ and $\delta\left(y-y_{i}\right)$ are Dirac delta functions. The force $p_{i}(t)$ has the following relation with the plate deflection $w\left(x_{i}, y_{i}, t\right)$ at point $\left(x_{i}, y_{i}\right)$ and the displacement $z_{i}(t)$ of the $i$ th mass as follows

$$
p_{i}(t)=-k_{i}\left[w\left(x_{i}, y_{i}, t\right)-z_{i}(t)\right]
$$

where $z_{i}$ should satisfy the following equation of motion

$$
m_{i} \frac{d^{2} z_{i}(t)}{d t^{2}}=-p_{i}(t), \quad i=1,2, \ldots, I
$$

Equations (1)-(5) form the governing equations of free vibration of the plate-spring-mass system. Considering free vibration of the system, the solutions of Eqs (1)-(5) have the following form:

$$
w=W(x, y) e^{j \omega t} ; \quad f(x, y, t)=F(x, y) e^{j \omega t} ; \quad p_{i}(t)=P_{i} e^{j \omega t} ; \quad z_{i}(t)=Z_{i} e^{j \omega t}
$$

where $\omega$ is the eigenfrequency of the plate-spring-mass system and $j=\sqrt{-1}$. Using the following dimensionless coordinates

$$
\xi=x / a ; \quad \eta=y / b
$$

Equation (1) can be written as

$$
\frac{1}{\beta^{4}} \frac{\partial^{4} W}{\partial \xi^{4}}+\frac{2}{\beta^{2}} \frac{\partial^{4} W}{\partial \xi^{2} \partial \eta^{2}}+\frac{\partial^{4} W}{\partial \eta^{4}}-\frac{\rho h}{D} b^{4} \omega^{2} W=\frac{b^{4}}{D} F(\xi, \eta)
$$

where $\beta=a / b$ is the aspect ratio of the plate. Similarly, Eqs (3)-(5) can be expressed as:

$$
\begin{aligned}
& F(\xi, \eta)=\frac{1}{a b} \sum_{i=1}^{I} P_{i} \delta\left(\xi-\xi_{i}\right) \delta\left(\eta-\eta_{i}\right) \\
& P_{i}=-k_{i}\left[W\left(\xi_{i}, \eta_{i}\right)-Z_{i}\right] ; \quad m_{i} \omega^{2} Z_{i}=P_{i}
\end{aligned}
$$

where $\xi_{i}=x_{i} / a$ and $\eta_{i}=y_{i} / b$. Equations (8)-(10) are the non-dimensional differential governing equations of free vibration of a thin plate attached to several sprung masses.

\section{Solutions}

For the plate with two opposite edges simply supported at $x=0$ and $a$, its solution can be given in the form of

$$
W(\xi, \eta)=\sum_{n=1}^{\infty} \sin (n \pi \xi) W_{n}(\eta)
$$


The above equation exactly satisfies the simply supported boundary conditions at edges $\xi=0$ and $\xi=1$. Expand $F(\xi, \eta)$ into a Fourier sinusoidal series about $\xi$ as follows

$$
F(\xi, \eta)=2 \sum_{n=1}^{\infty} \sin (n \pi \xi) F_{n}(\eta) ; \quad F_{n}(\eta)=\int_{0}^{1} F(\xi, \eta) \sin (n \pi \xi) d \xi
$$

It should be mentioned that the convergence error in the above equation has not been eliminated due to the Gibb's effect. Substituting Eqs (11) and (12) into Eq. (8) gives

$$
\frac{d^{4} W_{n}(\eta)}{d \eta^{4}}-2 \frac{(n \pi)^{2}}{\beta^{2}} \frac{d^{2} W_{n}(\eta)}{d \eta^{2}}+\left[\frac{(n \pi)^{4}}{\beta^{4}}-\alpha^{4}\right] W_{n}(\eta)=2 \frac{b^{4}}{D} F_{n}(\eta)
$$

where $\alpha^{4}=\rho h b^{4} \omega^{2} / D$. According to the theory of ordinary differential equations, the solution of Eq. (13) can be obtained. When $\alpha<n \pi / \beta$, one has

$$
W_{n}(\eta)=A_{n} \sinh \left(\lambda_{n 1} \eta\right)+B_{n} \cosh \left(\lambda_{n 1} \eta\right)+C_{n} \sinh \left(\lambda_{n 2} \eta\right)+D_{n} \cosh \left(\lambda_{n 2} \eta\right)+\tilde{W}_{n}(\eta)
$$

where

$$
\begin{aligned}
& \tilde{W}_{n}(\eta)=\frac{b^{4}}{D \alpha^{2} \lambda_{n 1} \lambda_{n 2}} \int_{0}^{\eta} F_{n}(s)\left[\lambda_{n 1} \sinh \left(\lambda_{n 2}(\eta-s)\right)-\lambda_{n 2} \sinh \left(\lambda_{n 1}(\eta-s)\right)\right] d s \\
& \lambda_{n 1}^{4}=\left[\frac{(n \pi)^{2}}{\beta^{2}}-\alpha^{2}\right]^{2} ; \quad \lambda_{n 2}^{4}=\left[\frac{(n \pi)^{2}}{\beta^{2}}+\alpha^{2}\right]^{2}
\end{aligned}
$$

When $\alpha>n \pi / \beta$, one has

$$
W_{n}(\eta)=A_{n} \sin \left(\lambda_{n 1} \eta\right)+B_{n} \cos \left(\lambda_{n 1} \eta\right)+C_{n} \sinh \left(\lambda_{n 2} \eta\right)+D_{n} \cosh \left(\lambda_{n 2} \eta\right)+\tilde{W}_{n}(\eta)
$$

where

$$
\tilde{W}_{n}(\eta)=\frac{b^{4}}{D \alpha^{2} \lambda_{n 1} \lambda_{n 2}} \int_{0}^{\eta} F_{n}(s)\left[\lambda_{n 1} \sinh \left(\lambda_{n 2}(\eta-s)\right)-\lambda_{n 2} \sin \left(\lambda_{n 1}(\eta-s)\right)\right] d s
$$

In Eqs (14a) and (16a), the first four terms with unknown constants, $A_{n}, B_{n}, C_{n}$ and $D_{n}$, are the homogeneous solutions while $\tilde{W}_{n}(\eta)$ is the special solution.

Assuming a positive integer $L$, when $n<L, \alpha>n \pi / \beta$ and when $n \geqslant L, \alpha<n \pi / \beta$, the mode shape $W(\xi, \eta)$ in Eq. (11) can be expressed in the form of

$$
\begin{aligned}
W(\xi, \eta)= & \sum_{n=1}^{L-1} \sin (n \pi \xi)\left\{A_{n} \sin \left(\lambda_{n 1} \eta\right)+B_{n} \cos \left(\lambda_{n 1} \eta\right)+C_{n} \sinh \left(\lambda_{n 2} \eta\right)+D_{n} \cosh \left(\lambda_{n 2} \eta\right)+\right. \\
& \left.\frac{b^{4}}{D \alpha^{2} \lambda_{n 1} \lambda_{n 2}} \int_{0}^{\eta} F_{n}(s)\left[\lambda_{n 1} \sinh \left(\lambda_{n 2}(\eta-s)\right)-\lambda_{n 2} \sin \left(\lambda_{n 1}(\eta-s)\right)\right] d s\right\}+ \\
& \sum_{n=L}^{\infty} \sin (n \pi \xi)\left\{A_{n} \sinh \left(\lambda_{n 1} \eta\right)+B_{n} \cosh \left(\lambda_{n 1} \eta\right)+C_{n} \sinh \left(\lambda_{n 2} \eta\right)+D_{n} \cosh \left(\lambda_{n 2} \eta\right)+\right. \\
& \left.\frac{b^{4}}{D \alpha^{2} \lambda_{n 1} \lambda_{n 2}} \int_{0}^{\eta} F_{n}(s)\left[\lambda_{n 1} \sinh \left(\lambda_{n 2}(\eta-s)\right)-\lambda_{n 2} \sinh \left(\lambda_{n 1}(\eta-s)\right)\right] d s\right\}
\end{aligned}
$$

Substituting Eq. (9) into Eq. (12) leads to

$$
F_{n}(\eta)=\frac{1}{a b} \sum_{i=1}^{I} P_{i} \sin \left(n \pi \xi_{i}\right) \delta\left(\eta-\eta_{i}\right)
$$

Substituting Eq. (18) into Eq. (17) gives 


$$
\begin{aligned}
W(\xi, \eta)= & \sum_{n=1}^{L-1} \sin (n \pi \xi)\left\{A_{n} \sin \left(\lambda_{n 1} \eta\right)+B_{n} \cos \left(\lambda_{n 1} \eta\right)+C_{n} \sinh \left(\lambda_{n 2} \eta\right)+D_{n} \cosh \left(\lambda_{n 2} \eta\right)+\right. \\
& \left.\frac{b^{2}}{\beta D \alpha^{2} \lambda_{n 1} \lambda_{n 2}} \sum_{i=1}^{I} P_{i} \sin \left(n \pi \xi_{i}\right)\left[\lambda_{n 1} \sinh \left(\lambda_{n 2}\left(\eta-\eta_{i}\right)\right)-\lambda_{n 2} \sin \left(\lambda_{n 1}\left(\eta-\eta_{i}\right)\right)\right] H\left(\eta-\eta_{i}\right)\right\}+ \\
& \sum_{n=L}^{\infty} \sin (n \pi \xi)\left\{A_{n} \sinh \left(\lambda_{n 1} \eta\right)+B_{n} \cosh \left(\lambda_{n 1} \eta\right)+C_{n} \sinh \left(\lambda_{n 2} \eta\right)+D_{n} \cosh \left(\lambda_{n 2} \eta\right)+\right. \\
& \left.\frac{b^{2}}{\beta D \alpha^{2} \lambda_{n 1} \lambda_{n 2}} \sum_{i=1}^{I} P_{i} \sin \left(n \pi \xi_{i}\right)\left[\lambda_{n 1} \sinh \left(\lambda_{n 2}\left(\eta-\eta_{i}\right)\right)-\lambda_{n 2} \sinh \left(\lambda_{n 1}\left(\eta-\eta_{i}\right)\right)\right] H\left(\eta-\eta_{i}\right)\right\}
\end{aligned}
$$

where

$$
H\left(\eta-\eta_{i}\right)=\left\{\begin{array}{l}
0 \eta<\eta_{i} \\
1 \eta \geqslant \eta_{i}
\end{array}\right. \text { is the Heaviside function. }
$$

\section{Boundary conditions}

Using the boundary conditions of the plate at the edges $\eta=0$ and $\eta=1$, the unknown constants $A_{n}, B_{n}, C_{n}$ and $D_{n}$ can be determined. Considering a general case: the plate is elastically restrained at the edges $\eta=0$ and $\eta=1$, the boundary conditions can be given by [20]:

$$
\begin{aligned}
& \frac{\partial^{2} W}{\partial \eta^{2}}+\nu \frac{\partial^{2} W}{\beta^{2} \partial \xi^{2}}=\frac{b k_{r 0}}{D} \frac{\partial W}{\partial \eta}, \text { at } \eta=0 \\
& \frac{\partial^{2} W}{\partial \eta^{2}}+\nu \frac{\partial^{2} W}{\beta^{2} \partial \xi^{2}}=-\frac{b k_{r 1}}{D} \frac{\partial W}{\partial \eta}, \text { at } \eta=1 ; \\
& \frac{\partial^{3} W}{\partial \eta^{3}}+(2-\nu) \frac{\partial^{3} W}{\beta^{2} \partial \eta \partial \xi^{2}}=-\frac{b^{3} k_{t 0}}{D} W, \text { at } \eta=0 ; \\
& \frac{\partial^{3} W}{\partial \eta^{3}}+(2-\nu) \frac{\partial^{3} W}{\beta^{2} \partial \eta \partial \xi^{2}}=\frac{b^{3} k_{t 1}}{D} W, \text { at } \eta=1
\end{aligned}
$$

where $k_{r 0}$ and $k_{r 1}$ are the stiffnesses of the rotational restraints at boundaries $y=0$ and $y=b$ respectively. $k_{t 0}$ and $k_{t 1}$ are the stiffnesses of the translational restraints at boundaries $y=0$ and $y=b$ respectively. By taking these stiffness coefficients to be infinite or zero, various classical boundary conditions can be obtained. For examples, if the plate is simply supported at $y=0$ and $y=b$, then $k_{r 0}=k_{r 1}=0$ and $k_{t 0}=k_{t 1}=\infty$. If the plate is clamped at $y=0$ and $y=b$, then $k_{r 0}=k_{r 1}=\infty$ and $k_{t 0}=k_{t 1}=\infty$. And if the plate is free at $y=0$ and $y=b$, then $k_{r 0}=k_{r 1}=0$ and $k_{t 0}=k_{t 1}=0$.

Substituting Eq. (19) into Eq. (20) gives the coefficients equations. When $n<L$

$$
\begin{aligned}
& -B_{n} \lambda_{n 1}^{2}+D_{n} \lambda_{n 2}^{2}-\frac{\nu(n \pi)^{2}}{\beta^{2}}\left(B_{n}+D_{n}\right)=K_{r 0}\left\{A_{n} \lambda_{n 1}+C_{n} \lambda_{n 2}\right\} \\
& -A_{n} \lambda_{n 1}^{2} \sin \lambda_{n 1}-B_{n} \lambda_{n 1}^{2} \cos \lambda_{n 1}+C_{n} \lambda_{n 2}^{2} \sinh \lambda_{n 2}+D_{n} \lambda_{n 2}^{2} \cosh \lambda_{n 2}+ \\
& \frac{b^{2}}{\beta D \alpha^{2}} \sum_{i=1}^{I} P_{i} \sin \left(n \pi \xi_{i}\right)\left[\lambda_{n 2} \sinh \left(\lambda_{n 2}\left(1-\eta_{i}\right)\right)+\lambda_{n 1} \sin \left(\lambda_{n 1}\left(1-\eta_{i}\right)\right)\right]- \\
& \frac{\nu(n \pi)^{2}}{\beta^{2}}\left\{A_{n} \sin \lambda_{n 1}+B_{n} \cos \lambda_{n 1}+C_{n} \sinh \lambda_{n 2}+D_{n} \cosh \lambda_{n 2}+\right.
\end{aligned}
$$


$\left.\frac{b^{2}}{\beta D \alpha^{2} \lambda_{n 1} \lambda_{n 2}} \sum_{i=1}^{I} P_{i} \sin \left(n \pi \xi_{i}\right)\left[\lambda_{n 1} \sinh \left(\lambda_{n 2}\left(1-\eta_{i}\right)\right)-\lambda_{n 2} \sin \left(\lambda_{n 1}\left(1-\eta_{i}\right)\right)\right]\right\}=$

$-K_{r 1}\left\{A_{n} \lambda_{n 1} \cos \lambda_{n 1}-B_{n} \lambda_{n 1} \sin \lambda_{n 1}+C_{n} \lambda_{n 2} \cosh \lambda_{n 2}+D_{n} \lambda_{n 2} \sinh \lambda_{n 2}+\right.$

$\left.\frac{b^{2}}{\beta D \alpha^{2}} \sum_{i=1}^{I} P_{i} \sin \left(n \pi \xi_{i}\right)\left[\cosh \left(\lambda_{n 2}\left(1-\eta_{i}\right)\right)-\cos \left(\lambda_{n 1}\left(1-\eta_{i}\right)\right)\right]\right\}$

$-A_{n} \lambda_{n 1}^{3}+C_{n} \lambda_{n 2}^{3}-\frac{(2-\nu)(n \pi)^{2}}{\beta^{2}}\left(A_{n} \lambda_{n 1}+C_{n} \lambda_{n 2}\right)=-K_{t 0}\left\{B_{n}+D_{n}\right\}$,

$-A_{n} \lambda_{n 1}^{3} \cos \lambda_{n 1}+B_{n} \lambda_{n 1}^{3} \sin \lambda_{n 1}+C_{n} \lambda_{n 2}^{3} \cosh \lambda_{n 2}+D_{n} \lambda_{n 2}^{3} \sinh \lambda_{n 2}+$

$\frac{b^{2}}{\beta D \alpha^{2}} \sum_{i=1}^{I} P_{i} \sin \left(n \pi \xi_{i}\right)\left[\lambda_{2 n}^{2} \cosh \left(\lambda_{n 2}\left(1-\eta_{i}\right)\right)+\lambda_{1 n}^{2} \cos \left(\lambda_{1 n}\left(1-\eta_{i}\right)\right)\right]-$

$\frac{(2-\nu)(n \pi)^{2}}{\beta^{2}}\left\{A_{n} \lambda_{n 1} \cos \lambda_{n 1}-B_{n} \lambda_{n 1} \sin \lambda_{n 1}+C_{n} \lambda_{n 2} \cosh \lambda_{n 2}+D_{n} \lambda_{n 2} \sinh \lambda_{n 2}+\right.$

$\left.\frac{b^{2}}{\beta D \alpha^{2}} \sum_{i=1}^{I} P_{i} \sin \left(n \pi \xi_{i}\right)\left[\cosh \left(\lambda_{n 2}\left(1-\eta_{i}\right)\right)-\cos \left(\lambda_{n 1}\left(1-\eta_{i}\right)\right)\right]\right\}=$

$K_{t 1}\left\{A_{n} \sin \lambda_{n 1}+B_{n} \cos \lambda_{n 1}+C_{n} \sinh \lambda_{n 2}+D_{n} \cosh \lambda_{n 2}+\right.$

$\left.\frac{b^{2}}{\beta D \alpha^{2} \lambda_{n 1} \lambda_{n 2}} \sum_{i=1}^{I} P_{i} \sin \left(n \pi \xi_{i}\right)\left[\lambda_{n 1} \sinh \left(\lambda_{n 2}\left(1-\eta_{i}\right)\right)-\lambda_{n 2} \sin \left(\lambda_{n 1}\left(1-\eta_{i}\right)\right)\right]\right\}$

when $n \geqslant L$

$B_{n} \lambda_{n 1}^{2}+D_{n} \lambda_{n 2}^{2}-\frac{\nu(n \pi)^{2}}{\beta^{2}}\left(B_{n}+D_{n}\right)=K_{r 0}\left\{A_{n} \lambda_{n 1}+C_{n} \lambda_{n 2}\right\}$,

$A_{n} \lambda_{n 1}^{2} \sinh \lambda_{n 1}+B_{n} \lambda_{n 1}^{2} \cosh \lambda_{n 1}+C_{n} \lambda_{n 2}^{2} \sinh \lambda_{n 2}+D_{n} \lambda_{n 2}^{2} \cosh \lambda_{n 2}+$

$\frac{b^{2}}{\beta D \alpha^{2}} \sum_{i=1}^{I} P_{i} \sin \left(n \pi \xi_{i}\right)\left[\lambda_{n 2} \sinh \left(\lambda_{n 2}\left(1-\eta_{i}\right)\right)-\lambda_{n 1} \sinh \left(\lambda_{n 1}\left(1-\eta_{i}\right)\right)\right]-$

$\frac{\nu(n \pi)^{2}}{\beta^{2}}\left\{A_{n} \sinh \lambda_{n 1}+B_{n} \cosh \lambda_{n 1}+C_{n} \sinh \lambda_{n 2}+D_{n} \cosh \lambda_{n 2}+\right.$

$\left.\frac{b^{2}}{\beta D \alpha^{2} \lambda_{n 1} \lambda_{n 2}} \sum_{i=1}^{I} P_{i} \sin \left(n \pi \xi_{i}\right)\left[\lambda_{n 1} \sinh \left(\lambda_{n 2}\left(1-\eta_{i}\right)\right)-\lambda_{n 2} \sinh \left(\lambda_{n 1}\left(1-\eta_{i}\right)\right)\right]\right\}=$

$-K_{r 1}\left\{A_{n} \lambda_{n 1} \cosh \lambda_{n 1}+B_{n} \lambda_{n 1} \sinh \lambda_{n 1}+C_{n} \lambda_{n 2} \cosh \lambda_{n 2}+D_{n} \lambda_{n 2} \sinh \lambda_{n 2}+\right.$

$\left.\frac{b^{2}}{\beta D \alpha^{2}} \sum_{i=1}^{I} P_{i} \sin \left(n \pi \xi_{i}\right)\left[\cosh \left(\lambda_{n 2}\left(1-\eta_{i}\right)\right)-\cosh \left(\lambda_{n 1}\left(1-\eta_{i}\right)\right)\right]\right\}$,

$A_{n} \lambda_{n 1}^{3}+C_{n} \lambda_{n 2}^{3}-\frac{(2-\nu)(n \pi)^{2}}{\beta^{2}}\left(A_{n} \lambda_{n 1}+C_{n} \lambda_{n 2}\right)=-K_{t 0}\left\{B_{n}+D_{n}\right\}$, 
$A_{n} \lambda_{n 1}^{3} \cosh \lambda_{n 1}+B_{n} \lambda_{n 1}^{3} \sinh \lambda_{n 1}+C_{n} \lambda_{n 2}^{3} \cosh \lambda_{n 2}+D_{n} \lambda_{n 2}^{3} \sinh \lambda_{n 2}+$

$\frac{b^{2}}{\beta D \alpha^{2}} \sum_{i=1}^{I} P_{i} \sin \left(n \pi \xi_{i}\right)\left[\lambda_{2 n}^{2} \cosh \left(\lambda_{n 2}\left(1-\eta_{i}\right)\right)-\lambda_{1 n}^{2} \cosh \left(\lambda_{1 n}\left(1-\eta_{i}\right)\right)\right]-$

$\frac{(2-\nu)(n \pi)^{2}}{\beta^{2}}\left\{A_{n} \lambda_{n 1} \cosh \lambda_{n 1}+B_{n} \lambda_{n 1} \sinh \lambda_{n 1}+C_{n} \lambda_{n 2} \cosh \lambda_{n 2}+D_{n} \lambda_{n 2} \sinh \lambda_{n 2}+\right.$

$\left.\frac{b^{2}}{\beta D \alpha^{2}} \sum_{i=1}^{I} P_{i} \sin \left(n \pi \xi_{i}\right)\left[\cosh \left(\lambda_{n 2}\left(1-\eta_{i}\right)\right)-\cosh \left(\lambda_{n 1}\left(1-\eta_{i}\right)\right)\right]\right\}=$

$K_{t 1}\left\{A_{n} \sinh \lambda_{n 1}+B_{n} \cosh \lambda_{n 1}+C_{n} \sinh \lambda_{n 2}+D_{n} \cosh \lambda_{n 2}+\right.$

$\left.\frac{b^{2}}{\beta D \alpha^{2} \lambda_{n 1} \lambda_{n 2}} \sum_{i=1}^{I} P_{i} \sin \left(n \pi \xi_{i}\right)\left[\lambda_{n 1} \sinh \left(\lambda_{n 2}\left(1-\eta_{i}\right)\right)-\lambda_{n 2} \sinh \left(\lambda_{n 1}\left(1-\eta_{i}\right)\right)\right]\right\}$

in which,

$$
K_{r 0}=\frac{b k_{r 0}}{D}, \quad K_{r 1}=\frac{b k_{r 1}}{D}, \quad K_{t 0}=\frac{b^{3} k_{t 0}}{D}, \quad K_{t 1}=\frac{b^{3} k_{t 1}}{D}
$$

From Eqs (21a) and (21b), the coefficients $A_{n}, B_{n}, C_{n}$ and $D_{n}$ can be solved and expressed as follows

$$
\begin{array}{ll}
A_{n}=\frac{b^{2}}{\beta D \alpha^{2}} \sum_{i=1}^{I} P_{i} \sin \left(n \pi \xi_{i}\right) q_{n i}^{1}, & B_{n}=\frac{b^{2}}{\beta D \alpha^{2}} \sum_{i=1}^{I} P_{i} \sin \left(n \pi \xi_{i}\right) q_{n i}^{2}, \\
C_{n}=\frac{b^{2}}{\beta D \alpha^{2}} \sum_{i=1}^{I} P_{i} \sin \left(n \pi \xi_{i}\right) q_{n i}^{3}, & D_{n}=\frac{b^{2}}{\beta D \alpha^{2}} \sum_{i=1}^{I} P_{i} \sin \left(n \pi \xi_{i}\right) q_{n i}^{4}
\end{array}
$$

$q_{n i}^{s}(s=1,2,3,4)$ are given in the Appendix.

\section{Eigenfrequency equation}

Substituting Eq. (23) into Eq. (19), the following displacement function is obtained

$$
\begin{aligned}
& W(\xi, \eta)=\frac{b^{2}}{\beta D \alpha^{2}} \sum_{i=1}^{I} P_{i}\left\{\sum _ { n = 1 } ^ { L - 1 } \operatorname { s i n } ( n \pi \xi _ { i } ) \operatorname { s i n } ( n \pi \xi ) \left\{q_{n i}^{1} \sin \left(\lambda_{n 1} \eta\right)+q_{n i}^{2} \cos \left(\lambda_{n 1} \eta\right)+\right.\right. \\
& \left.q_{n i}^{3} \sinh \left(\lambda_{n 2} \eta\right)+q_{n i}^{4} \cosh \left(\lambda_{n 2} \eta\right)+\left[\frac{1}{\lambda_{n 2}} \sinh \left(\lambda_{n 2}\left(\eta-\eta_{i}\right)\right)-\frac{1}{\lambda_{n 1}} \sin \left(\lambda_{n 1}\left(\eta-\eta_{i}\right)\right)\right] H\left(\eta-\eta_{i}\right)\right\}+ \\
& \sum_{n=L}^{\infty} \sin \left(n \pi \xi_{i}\right) \sin (n \pi \xi)\left\{q_{n i}^{1} \sinh \left(\lambda_{n 1} \eta\right)+q_{n i}^{2} \cosh \left(\lambda_{n 1} \eta\right)+q_{n i}^{3} \sinh \left(\lambda_{n 2} \eta\right)+\right. \\
& \left.\left.q_{n i}^{4} \cosh \left(\lambda_{n 2} \eta\right)+\left[\frac{1}{\lambda_{n 2}} \sinh \left(\lambda_{n 2}\left(\eta-\eta_{i}\right)\right)-\frac{1}{\lambda_{n 1}} \sinh \left(\lambda_{n 1}\left(\eta-\eta_{i}\right)\right)\right] H\left(\eta-\eta_{i}\right)\right\}\right\}
\end{aligned}
$$

From Eq. (10), one has

$$
\left(1-\frac{k_{i}}{m_{i} \omega^{2}}\right) P_{i}+k_{i} W\left(\xi_{i}, \eta_{i}\right)=0, i=1,2, \ldots, I
$$


Substituting Eq. (24) into the above equation, a group of homogeneous equations about $P_{i}(i=1,2, \ldots, I)$ is obtained as follows

$$
\left[\begin{array}{cccc}
B_{11} & B_{12} & \cdots & B_{1 I} \\
B_{21} & B_{22} & \cdots & B_{2 I} \\
\vdots & \vdots & \ddots & \vdots \\
B_{I 1} & B_{I 2} & \cdots & B_{I I}
\end{array}\right]\left\{\begin{array}{c}
P_{1} \\
P_{2} \\
\vdots \\
P_{I}
\end{array}\right\}=\left\{\begin{array}{c}
0 \\
0 \\
\vdots \\
0
\end{array}\right\}
$$

in which,

$$
\begin{aligned}
& B_{i j}=\sum_{n=1}^{L-1} \sin \left(n \pi \xi_{i}\right) \sin \left(n \pi \xi_{j}\right)\left\{q_{n i}^{1} \sin \left(\lambda_{n 1} \eta_{j}\right)+q_{n i}^{2} \cos \left(\lambda_{n 1} \eta_{j}\right)+q_{n i}^{3} \sinh \left(\lambda_{n 2} \eta_{j}\right)+\right. \\
& \left.q_{n i}^{4} \cosh \left(\lambda_{n 2} \eta_{j}\right)+\left[\frac{1}{\lambda_{n 2}} \sinh \left(\lambda_{n 2}\left(\eta_{j}-\eta_{i}\right)\right)-\frac{1}{\lambda_{n 1}} \sin \left(\lambda_{n 1}\left(\eta_{j}-\eta_{i}\right)\right)\right] H\left(\eta_{j}-\eta_{i}\right)\right\}+ \\
& \sum_{n=L}^{\infty} \sin \left(n \pi \xi_{i}\right) \sin \left(n \pi \xi_{j}\right)\left\{q_{n i}^{1} \sinh \left(\lambda_{n 1} \eta_{j}\right)+q_{n i}^{2} \cosh \left(\lambda_{n 1} \eta_{j}\right)+q_{n i}^{3} \sinh \left(\lambda_{n 2} \eta_{j}\right)+\right. \\
& \left.q_{n i}^{4} \cosh \left(\lambda_{n 2} \eta_{j}\right)+\left[\frac{1}{\lambda_{n 2}} \sinh \left(\lambda_{n 2}\left(\eta_{j}-\eta_{i}\right)\right)-\frac{1}{\lambda_{n 1}} \sinh \left(\lambda_{n 1}\left(\eta_{j}-\eta_{i}\right)\right)\right] H\left(\eta_{j}-\eta_{i}\right)\right\}, \quad i \neq j \\
& B_{i i}=\sum_{n=1}^{L-1} \sin ^{2}\left(n \pi \xi_{i}\right)\left\{q_{n i}^{1} \sin \left(\lambda_{n 1} \eta_{i}\right)+q_{n i}^{2} \cos \left(\lambda_{n 1} \eta_{i}\right)+q_{n i}^{3} \sinh \left(\lambda_{n 2} \eta_{i}\right)+q_{n i}^{4} \cosh \left(\lambda_{n 2} \eta_{i}\right)+\right. \\
& \sum_{n=L}^{\infty} \sin ^{2}\left(n \pi \xi_{i}\right)\left\{q_{n i}^{1} \sinh \left(\lambda_{n 1} \eta_{i}\right)+q_{n i}^{2} \cosh \left(\lambda_{n 1} \eta_{i}\right)+q_{n i}^{3} \sinh \left(\lambda_{n 2} \eta_{i}\right)+\right. \\
& q_{n i}^{4} \cosh \left(\lambda_{n 2} \eta_{i}\right)+\frac{\alpha^{2}}{\tau_{j}}-\frac{1}{\alpha^{2} \gamma_{j}}
\end{aligned}
$$

in which,

$$
\tau_{j}=\frac{k_{j} b^{2}}{\beta D} ; \quad \gamma_{j}=\frac{m_{j}}{\rho h a b}
$$

where $\tau_{j}$ and $\gamma_{j}$ are referred to as the stiffness ratio and mass ratio. In order to obtain non-zero solutions about $P_{i}$ $(i=1,2, \ldots, I)$, the coefficient determinant of Eq. (25) should be zero, i.e.

$$
\left|\begin{array}{cccc}
B_{11} & B_{12} & \cdots & B_{1 I} \\
B_{21} & B_{22} & \cdots & B_{2 I} \\
\vdots & \vdots & \ddots & \vdots \\
B_{I 1} & B_{I 2} & \cdots & B_{I I}
\end{array}\right|=0
$$

It can be seen from Eq. (29) that the order of the eigenvalue equations is equal to the number of attached sprung masses. The equation is non-linear in respect to eigenfrequency $\omega$ which can be determined using the root searching method. Substituting the known values of $\omega$ into Eq. (26) yields the solution of coefficient vector $\{P\}$. Then, substituting these results into Eq. (24) gives the mode shapes of the plate.

From Eq. (24) that if $W\left(\xi_{j}, \eta_{j}\right)=0$ for a mode, then $P_{j}=0$, thus the $j$ th sprung mass has no effect on that mode. Similarly, if $W\left(\xi_{i}, \eta_{i}\right)=0(i=1,2, \ldots, I)$ for a mode, then $P_{i}=0(i=1,2, \ldots, I)$, which indicates that the mode is independent of all the sprung masses. Moreover, it is well known that if a bare plate has duplicate eigenfrequencies, an arbitrary linear combination of the two modes is still the mode of the same eigenfrequency [21]. Therefore, one can always find such a coefficient that the combination mode is equal to zero at an arbitrarily given point, i.e. the node of the combination mode can be arranged at an arbitrary location of the plate. When such a plate is attached to one 
Table 1

The convergence study of the first five coupled eigenfrequencies for a SSSS square plate with one sprung mass in respect to the number of terms $(n)$ used

\begin{tabular}{|c|c|c|c|c|c|c|c|}
\hline$\xi_{1}, \eta_{1}$ & $\tau_{1}, \quad \gamma_{1}$ & $n$ & $\Omega_{1}$ & $\Omega_{2}$ & $\Omega_{3}$ & $\Omega_{4}$ & $\Omega_{5}$ \\
\hline \multirow[t]{12}{*}{$0.5,0.5$} & $100.0,0.1$ & 10 & 15.81 & 36.93 & 102.99 & 178.81 & 258.18 \\
\hline & & 20 & 15.81 & 36.89 & 102.98 & 178.81 & 258.18 \\
\hline & & 30 & 15.81 & 36.88 & 102.97 & 178.81 & 258.17 \\
\hline & & 40 & 15.81 & 36.88 & 102.97 & 178.80 & 258.17 \\
\hline & $500,0.2$ & 10 & 14.23 & 51.68 & 117.57 & 183.44 & 264.26 \\
\hline & & 20 & 14.23 & 51.51 & 117.34 & 183.35 & 264.15 \\
\hline & & 30 & 14.23 & 51.48 & 117.29 & 183.34 & 264.13 \\
\hline & & 40 & 14.23 & 51.47 & 117.28 & 183.33 & 264.12 \\
\hline & $2500,0.5$ & 10 & 10.98 & 55.87 & 139.09 & 197.20 & 282.77 \\
\hline & & 20 & 10.98 & 55.58 & 138.30 & 196.46 & 281.77 \\
\hline & & 30 & 10.98 & 55.53 & 138.16 & 196.33 & 281.66 \\
\hline & & 40 & 10.98 & 55.51 & 138.11 & 196.29 & 281.54 \\
\hline \multirow[t]{12}{*}{$0.6,0.7$} & $100,0.1$ & 10 & 16.84 & 31.58 & 55.02 & 79.93 & 99.42 \\
\hline & & 20 & 16.84 & 31.55 & 55.00 & 79.92 & 99.42 \\
\hline & & 30 & 16.84 & 31.55 & 55.00 & 79.92 & 99.42 \\
\hline & & 40 & 16.84 & 31.55 & 55.00 & 79.92 & 99.42 \\
\hline & $500,0.2$ & 10 & 15.51 & 35.64 & 67.27 & 84.69 & 103.05 \\
\hline & & 20 & 15.51 & 35.59 & 67.15 & 84.60 & 102.98 \\
\hline & & 30 & 15.51 & 35.58 & 67.13 & 84.58 & 102.96 \\
\hline & & 40 & 15.51 & 35.58 & 67.12 & 84.58 & 102.96 \\
\hline & $2500,0.5$ & 10 & 12.33 & 34.22 & 71.31 & 89.97 & 111.22 \\
\hline & & 20 & 12.32 & 34.16 & 71.19 & 89.82 & 110.88 \\
\hline & & 30 & 12.32 & 34.15 & 71.17 & 89.79 & 110.82 \\
\hline & & 40 & 12.32 & 34.15 & 71.16 & 89.78 & 110.80 \\
\hline
\end{tabular}

sprung mass, the separation of the duplicate eigenfrequencies will inevitably occur. In other words, one of the two eigenfrequencies will hold the line $\left(P_{1}=0\right)$, while the other varies with the parameters of the system $\left(P_{1} \neq 0\right)$, such as the attached position of the sprung mass, the mass ratio and the stiffness ratio. Taking a fully simply supported square plate as an example, the $(1,3)$ mode $\sin (\pi x / a) \sin (3 \pi y / b)$ and the $(3,1)$ mode $\sin (3 \pi x / a) \sin (\pi y / b)$ are both the double symmetric modes and have the same eigenfrequency. Whether a sprung mass is attached on any position of the plate, one always obtains an invariable eigenfrequency $\alpha^{2}=10 \pi^{2}$.

The invariable eigenfrequencies cannot be predicted by the present root searching method because the coefficient vector $\{P\}$ equals zero. However, each invariable mode can be judged by observing the modal shapes of the bare plate and the attached positions of the sprung masses. For an invariable mode, all the sprung masses are on the nodes of the modal shape of the bare plate.

The solution provided in the paper is general and includes several special cases when taking the stiffness ratio $\tau_{j}$ and/or the mass ratios $\gamma_{j}$ to their extreme values, such as

1. When $\tau_{j}=\infty$, a rigid mass is attached at $\left(x_{j}, y_{j}\right)$ on the plate;

2. When $\gamma_{j}=\infty$, an elastic point-support is provided at $\left(x_{j}, y_{j}\right)$ on the plate and

3. When $\tau_{j}=\infty$ and $\gamma_{j}=\infty$, a rigid vertical support is given at $\left(x_{j}, y_{j}\right)$ on the plate.

\section{Numerical studies}

To demonstrate the accuracy and applicability of the present approach, numerical calculations have been conducted. Only the results concerned with the coupled vibration (where the sprung masses have effect on the plate vibration) are presented and discussed. The Poisson's ratio is taken to be $\nu=0.3$ and $10^{10}$ is used to simulate a rigid support in the calculations.

\subsection{Convergence study}

The first problem considered is a simply supported (SSSS) square plate with one sprung mass. Three groups of parameters of the sprung mass are used in the computations respectively: $\tau_{1}=100, \gamma_{1}=0.1 ; \tau_{1}=500$, 
Table 2

The convergence study of the first five coupled eigenfrequencies for square plates with two sprung masses in respect to the number of terms $(n)$ used

\begin{tabular}{|c|c|c|c|c|c|c|}
\hline B.C. & $n$ & $\Omega_{1}$ & $\Omega_{2}$ & $\Omega_{3}$ & $\Omega_{4}$ & $\Omega_{5}$ \\
\hline \multicolumn{7}{|c|}{$\begin{array}{l}\xi_{1}=\eta_{1}=0.4, \xi_{2}=0.7, \eta_{2}=0.6, \tau_{1}=\tau_{2}=200 \\
\gamma_{1}=0.1, \gamma_{2}=0.2\end{array}$} \\
\hline \multirow[t]{4}{*}{ SSSS } & 10 & 13.91 & 26.29 & 46.89 & 51.27 & 62.90 \\
\hline & 20 & 13.91 & 26.25 & 46.84 & 51.22 & 62.83 \\
\hline & 30 & 13.91 & 26.25 & 46.83 & 51.22 & 62.82 \\
\hline & 40 & 13.91 & 26.25 & 46.83 & 51.21 & 62.81 \\
\hline \multirow[t]{4}{*}{ SFSF* } & 10 & 8.02 & 16.79 & 25.04 & 34.77 & 42.29 \\
\hline & 20 & 8.02 & 16.79 & 25.01 & 34.74 & 42.27 \\
\hline & 30 & 8.02 & 16.79 & 25.01 & 34.74 & 42.27 \\
\hline & 40 & 8.02 & 16.79 & 25.01 & 34.74 & 42.27 \\
\hline \multicolumn{7}{|c|}{$\begin{array}{l}\xi_{1}=\eta_{1}=0.25, \xi_{2}=\eta_{2}=0.5, \tau_{1}=100, \tau_{2}=50 \\
\gamma_{1}=\gamma_{2}=0.1\end{array}$} \\
\hline \multirow[t]{4}{*}{ SSSS } & 10 & 14.49 & 24.87 & 31.64 & 54.04 & 81.81 \\
\hline & 20 & 14.49 & 24.86 & 31.63 & 54.03 & 81.80 \\
\hline & 30 & 14.49 & 24.85 & 31.62 & 54.03 & 81.80 \\
\hline & 40 & 14.49 & 24.85 & 31.62 & 54.03 & 81.80 \\
\hline \multirow[t]{4}{*}{ SFSF $^{*}$} & 10 & 8.40 & 16.43 & 22.12 & 29.71 & 41.93 \\
\hline & 20 & 8.40 & 16.43 & 22.10 & 29.69 & 41.92 \\
\hline & 30 & 8.40 & 16.43 & 22.10 & 29.69 & 41.92 \\
\hline & 40 & 8.40 & 16.43 & 22.10 & 29.69 & 41.92 \\
\hline
\end{tabular}

${ }^{*}$ Two opposite edges are simply supported and the two other edges are free.

$\gamma_{1}=0.2$ and $\tau_{1}=2500, \gamma_{1}=0.5$. Two different locations of the sprung mass are considered: $\xi_{1}=0.5, \eta_{1}=0.5$ and $\xi_{1}=0.6, \eta_{1}=0.7$. The convergence corresponding to different terms of the series for the first five coupled eigenfrequencies $\Omega_{i}=\alpha_{i}^{2}=\omega_{i} b^{2} \sqrt{\rho h / D}(i=1,2, \ldots, 5)$ is shown in Table 1.

It can be seen from Table 1 that the calculations converge quickly for all of the studied cases. With the increase of the number of terms, all the eigenfrequencies monotonically and steadily decrease. Therefore, the results obtained are the upper bounds of the exact values, similar to those obtained from the use of the Rayleigh-Ritz method. Taking only 20 terms, the maximum difference relative to those using 40 terms is less than $0.2 \%$ for the studied cases. The CPU time to use 40 terms to search the first five eigenvalues is less than two seconds on a Pentium 4 microcomputer. It is observed that the computation converges slightly slower when the sprung mass is placed at the centre of the plate than when it is away from the centre of the plate. When the mass and stiffness ratios increase, more terms are needed to maintain the same accuracy. However, the differences between the convergence rates for the studied cases are small.

Table 2 compares the convergence of the eigenvalues of square plates attached with two sprung masses. Two kinds of boundary conditions and two locations of the sprung masses are considered. Compared with the results in Table 1, it is seen from Table 2 that a better convergence is achieved. Therefore, in the following calculations, 40 terms are taken for all the cases.

\subsection{Comparative study}

A comparative study is given in Table 3 for a simply supported rectangular plate with one sprung mass. The solutions from the eigenfunction expansion (EE) method presented by Avalos et al. [10] and Wu et al. [12] and the solutions from the finite element (FE) method presented by Wu et al. [12] are taken as the reference. Moreover, the solutions from the Lagrangian multiplier (LM) method presented by Kim and Dickinson [3] are taken for comparison for the case of a rigid vertical point-support.

Table 3 shows that the present solutions agree well with those obtained from the finite element solutions and with most of those from the eigenfunction expansion solutions. However, almost all the answers from the present method are smaller or equal to those quoted in literature. 
Table 3

Comparison of the first five coupled eigenfrequencies with published data (SSSS rectangular plates with one sprung mass)

\begin{tabular}{|c|c|c|c|c|c|c|}
\hline Parameters & Methods & $\Omega_{1}$ & $\Omega_{2}$ & $\Omega_{3}$ & $\Omega_{4}$ & $\Omega_{5}$ \\
\hline $\begin{array}{l}\tau_{1}=3.375, \gamma_{1}=0.5 \\
\beta=2 / 3, \xi_{1}=0.75, \eta_{1}=0.75\end{array}$ & $\begin{array}{l}\text { Present } \\
\text { FE [12] }\end{array}$ & $\begin{array}{l}2.588 \\
2.588\end{array}$ & $\begin{array}{l}32.129 \\
32.937\end{array}$ & $\begin{array}{l}61.740 \\
63.743\end{array}$ & $\begin{array}{l}98.730 \\
99.844\end{array}$ & $\begin{array}{l}111.048 \\
114.314\end{array}$ \\
\hline $\begin{array}{l}\tau_{1}=0.0625, \gamma_{1}=0.25 \\
\beta=2, \xi_{1}=0.75, \eta_{1}=0.75\end{array}$ & $\begin{array}{l}\text { Present } \\
\text { FE [12] } \\
\text { EE [12] } \\
\text { EE [10] }\end{array}$ & $\begin{array}{l}0.500 \\
0.500 \\
0.500 \\
0.500\end{array}$ & $\begin{array}{l}12.340 \\
12.655 \\
12.339 \\
12.340\end{array}$ & $\begin{array}{l}19.742 \\
20.697 \\
19.742 \\
19.742\end{array}$ & $\begin{array}{l}32.077 \\
33.812 \\
32.077 \\
32.077\end{array}$ & $\begin{array}{l}41.947 \\
42.318 \\
41.947 \\
41.947\end{array}$ \\
\hline $\begin{array}{l}\tau_{1}=0.125, \gamma_{1}=0.5 \\
\beta=2, \xi_{1}=0.75, \eta_{1}=0.75\end{array}$ & $\begin{array}{l}\text { Present } \\
\text { FE [12] } \\
\text { EE [12] } \\
\text { EE [10] }\end{array}$ & $\begin{array}{l}0.499 \\
0.499 \\
0.499 \\
0.499\end{array}$ & $\begin{array}{l}12.342 \\
12.658 \\
12.342 \\
12.342\end{array}$ & $\begin{array}{l}19.746 \\
20.700 \\
19.745 \\
19.746\end{array}$ & $\begin{array}{l}32.078 \\
33.813 \\
32.078 \\
32.078\end{array}$ & $\begin{array}{l}41.949 \\
42.319 \\
41.948 \\
41.949\end{array}$ \\
\hline $\begin{array}{l}\tau_{1}=5, \gamma_{1}=0.1 \\
\beta=1, \xi_{1}=0.75, \eta_{1}=0.5 \\
\tau_{1}=5, \gamma_{1}=1 \\
\beta=1, \xi_{1}=0.5, \eta_{1}=0.5\end{array}$ & $\begin{array}{l}\text { Present } \\
\text { EE [10] } \\
\text { Present } \\
\text { EE [10] }\end{array}$ & $\begin{array}{l}6.923 \\
6.930 \\
2.173 \\
2.174\end{array}$ & $\begin{array}{l}20.022 \\
20.022 \\
20.242 \\
20.243\end{array}$ & $\begin{array}{l}49.554 \\
49.555 \\
98.898 \\
98.899\end{array}$ & $\begin{array}{r}98.798 \\
98.798 \\
177.709 \\
177.709\end{array}$ & 128.383 \\
\hline $\begin{array}{l}\tau_{1}=\infty, \gamma_{1}=1 \\
\beta=1, \xi_{1}=0.5, \eta_{1}=0.5\end{array}$ & $\begin{array}{l}\text { Present } \\
\text { EE [10] }\end{array}$ & $\begin{array}{l}8.474 \\
8.493\end{array}$ & $\begin{array}{l}56.310 \\
57.175\end{array}$ & $\begin{array}{l}148.089 \\
150.570\end{array}$ & $\begin{array}{l}208.663 \\
212.787\end{array}$ & 298.017 \\
\hline $\begin{array}{l}\tau_{1}=\infty, \gamma_{1}=0.5 \\
\beta=1, \xi_{1}=0.75, \eta_{1}=0.75\end{array}$ & $\begin{array}{l}\text { Present } \\
\text { EE [10] }\end{array}$ & $\begin{array}{l}14.779 \\
14.892\end{array}$ & $\begin{array}{l}29.898 \\
30.437\end{array}$ & $\begin{array}{l}64.646 \\
65.466\end{array}$ & $\begin{array}{l}92.758 \\
93.333\end{array}$ & 115.406 \\
\hline $\begin{array}{l}\tau_{1}=\infty, \gamma_{1}=0.5 \\
\beta=2, \xi_{1}=0.75, \eta_{1}=0.5\end{array}$ & $\begin{array}{l}\text { Present } \\
\text { EE [10] }\end{array}$ & $\begin{array}{l}7.343 \\
7.428\end{array}$ & $\begin{array}{l}15.426 \\
15.506\end{array}$ & $\begin{array}{l}29.105 \\
29.347\end{array}$ & $\begin{array}{l}54.658 \\
68.003\end{array}$ & $\begin{array}{l}76.608 \\
93.760\end{array}$ \\
\hline $\begin{array}{l}\tau_{1}=\infty, \gamma_{1}=\infty \\
\beta=1, \xi_{1}=0.5, \eta_{1}=0.5\end{array}$ & $\begin{array}{l}\text { Present } \\
\text { LM [3] }\end{array}$ & $\begin{array}{l}52.631 \\
53.170\end{array}$ & $\begin{array}{l}146.700 \\
148.201\end{array}$ & 207.679 & 297.446 & 405.937 \\
\hline
\end{tabular}

\subsection{Parametric study}

Consider a square plate with one sprung mass. The plate has two opposite edges simply supported and the two other edges clamped (SCSC). Table 4 gives the first six eigenfrequencies of the coupled vibration with respect to the mass ratio $\gamma_{1}$ and stiffness ratio $\tau_{1}$. Three different locations of the sprung mass are considered, i.e. $\left(\xi_{1}=0.5 ; \eta_{1}=0.5\right)$, $\left(\xi_{1}=0.5 ; \eta_{1}=0.25\right)$ and $\left(\xi_{1}=0.25 ; \eta_{1}=0.25\right)$. It can be seen from Table 4 that increasing the mass ratio $\gamma_{1}$ will decrease the eigenfrequencies, while increasing the stiffness ratio will increase the eigenfrequencies. Moreover, if a given finite mass is placed at the centre of the plate, it will lead to the smallest fundamental eigenfrequency; while an elastic point-support located at the centre of the plate provides the largest fundamental eigenfrequency.

Consider a simply supported square plate attached with one sprung mass at its centre. Figures 2-4 give the first four eigenfrequencies of coupled vibration with respect to the stiffness ratio $\tau_{1}$ when the mass ratio $\gamma_{1}$ is equal to 0.5 , 5 and infinity. The parameter $\tau_{1}$ is plotted along a logarithmic axis. It is shown that with the increase of the stiffness of the sprung mass, all of the eigenfrequencies monotonically increase. It is seen from Figs 2 and 3 that the variations of the eigenfrequencies increase with increase of $\tau_{1}$ for moderate spring stiffness. The stiffness of the sprung mass, which results in the steep variation of the eigenfrequencies, increases with the order of the eigenfrequencies. It can be seen that the first eigenfrequency is sensitive in the range of $5<\tau_{1}<100$; the second eigenfrequency is sensitive within $50<\tau_{1}<500$, the third within $100<\tau_{1}<10000$ and the fourth within $500<\tau_{1}<10000$. Thus when the stiffness of the sprung mass is small, the coupled vibration between the plate and the sprung mass is rather weak because the natural frequency of the sprung mass is much lower than the natural frequencies of the bare plate. However, when the stiffness of the sprung mass is very large, the sprung mass acts as a rigid mass attached on the plate. Figure 4 shows that the smaller the stiffness of the internal support, the smaller the effect of the elastic point-support on the eigenfrequencies of the plate and the large internal stiffness acts as a rigid point support. It should be mentioned that all of the coupled modes in Figs $2-4$ are double symmetric because the sprung mass is just placed at the centre of the plate. Correspondingly, all of the non symmetric-symmetric modes belong to the invariable modes.

Table 5 studies the effect of boundary conditions on the coupled eigenfrequencies of the square plate. The plate is simply supported along all edges. However, rotational springs with the same stiffness are added to two opposite 
Table 4

The first six eigenfrequencies of coupled vibration for a SCSC square plate with one sprung mass

\begin{tabular}{|c|c|c|c|c|c|c|}
\hline$\gamma_{1}, \tau_{1}$ & $\Omega_{1}$ & $\Omega_{2}$ & $\Omega_{3}$ & $\Omega_{4}$ & $\Omega_{5}$ & $\Omega_{6}$ \\
\hline \multicolumn{7}{|c|}{$\xi_{1}=0.5, \quad \eta_{1}=0.5$} \\
\hline $0.1,100$ & 20.728 & $41.679^{\circ}$ & 104.474 & 130.814 & 200.882 & 259.462 \\
\hline $0.5,100$ & 10.555 & 36.687 & 104.320 & 130.730 & 200.861 & 259.452 \\
\hline $0.5,1000$ & 14.198 & 59.083 & 112.901 & 145.768 & 209.107 & 266.006 \\
\hline $0.1, \infty$ & 23.415 & 83.600 & 119.593 & 177.645 & 233.957 & 286.155 \\
\hline $0.5, \infty$ & 14.794 & 69.702 & 117.209 & 169.783 & 228.676 & 282.962 \\
\hline$\infty, 100$ & 35.763 & 104.284 & 130.710 & 200.856 & 259.449 & 307.965 \\
\hline$\infty, 1000$ & 54.068 & 112.328 & 144.591 & 208.772 & 265.838 & 313.127 \\
\hline$\infty, \infty$ & 64.003 & 116.541 & 167.428 & 227.307 & 282.160 & 328.076 \\
\hline \multicolumn{7}{|c|}{$\xi_{1}=0.5, \quad \eta_{1}=0.25$} \\
\hline $0.1,100$ & 23.775 & 34.920 & 72.556 & 103.091 & 130.403 & 141.976 \\
\hline $0.5,100$ & 11.892 & 31.468 & 72.109 & 103.028 & 130.350 & 141.899 \\
\hline $0.5,1000$ & 18.222 & 39.587 & 82.565 & 106.993 & 133.588 & 159.052 \\
\hline $0.1, \infty$ & 26.640 & 56.464 & 93.804 & 113.806 & 135.033 & 184.900 \\
\hline $0.5, \infty$ & 19.384 & 43.339 & 88.249 & 110.396 & 134.584 & 180.147 \\
\hline$\infty, 100$ & 31.066 & 72.014 & 103.014 & 130.337 & 141.881 & 200.624 \\
\hline$\infty, 1000$ & 36.169 & 81.282 & 106.623 & 133.485 & 157.987 & 205.464 \\
\hline$\infty, \infty$ & 38.629 & 86.539 & 109.641 & 134.471 & 178.680 & 207.124 \\
\hline \multicolumn{7}{|c|}{$\xi_{1}=0.25, \quad \eta_{1}=0.25$} \\
\hline $0.1,100$ & 24.927 & 32.633 & 56.316 & 70.963 & 96.785 & 102.825 \\
\hline $0.5,100$ & 12.197 & 30.193 & 55.993 & 70.727 & 96.620 & 102.768 \\
\hline $0.5,1000$ & 19.722 & 34.476 & 58.985 & 75.675 & 100.454 & 110.916 \\
\hline $0.1, \infty$ & 27.649 & 48.201 & 63.207 & 82.271 & 101.093 & 120.601 \\
\hline $0.5, \infty$ & 21.221 & 36.662 & 60.133 & 78.208 & 100.864 & 117.205 \\
\hline$\infty, 100$ & 29.983 & 55.931 & 70.678 & 96.582 & 102.755 & 129.761 \\
\hline$\infty, 1000$ & 32.286 & 58.472 & 75.014 & 100.355 & 110.100 & 132.514 \\
\hline$\infty, \infty$ & 33.333 & 59.438 & 77.240 & 100.794 & 116.199 & 134.110 \\
\hline
\end{tabular}

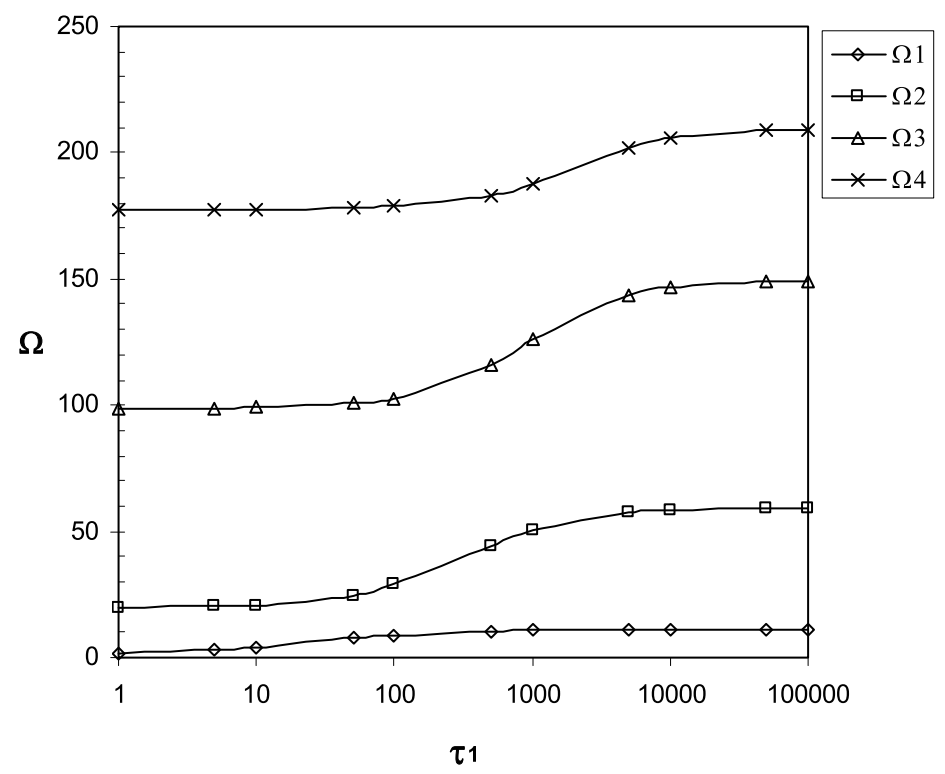

Fig. 2. The first four coupled eigenfrequencies of a SSSS square plate with one sprung mass at its centre $\left(\xi_{1}=\eta_{1}=0.5\right)$, with respect to the stiffness ratio $\tau_{1}$ when the mass ratio $\gamma_{1}$ is equal to 0.5 .

edges. In the present investigation, two cases are considered: the plate with one sprung mass and the plate attached with two sprung masses. It can be seen from Table 5 that increasing the stiffness of the rotational springs does not always lead to increases of the coupled eigenfrequencies. 


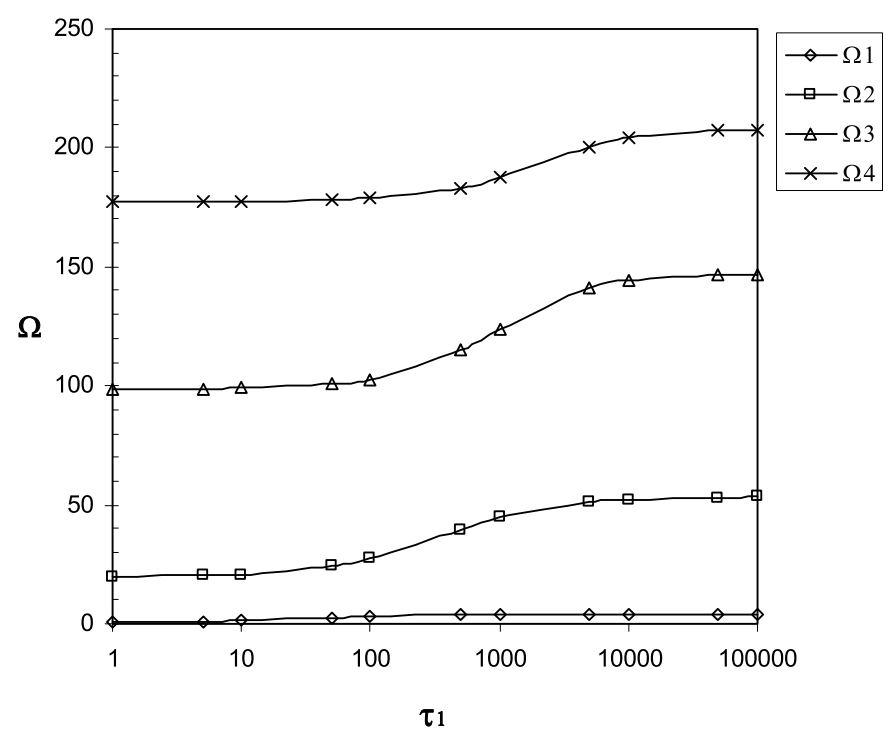

Fig. 3. The first four coupled eigenfrequencies of a SSSS square plate with one sprung mass at its centre $\left(\xi_{1}=\eta_{1}=0.5\right)$, with respect to the stiffness ratio $\tau_{1}$ when the mass ratio $\gamma_{1}$ is equal to 5 .

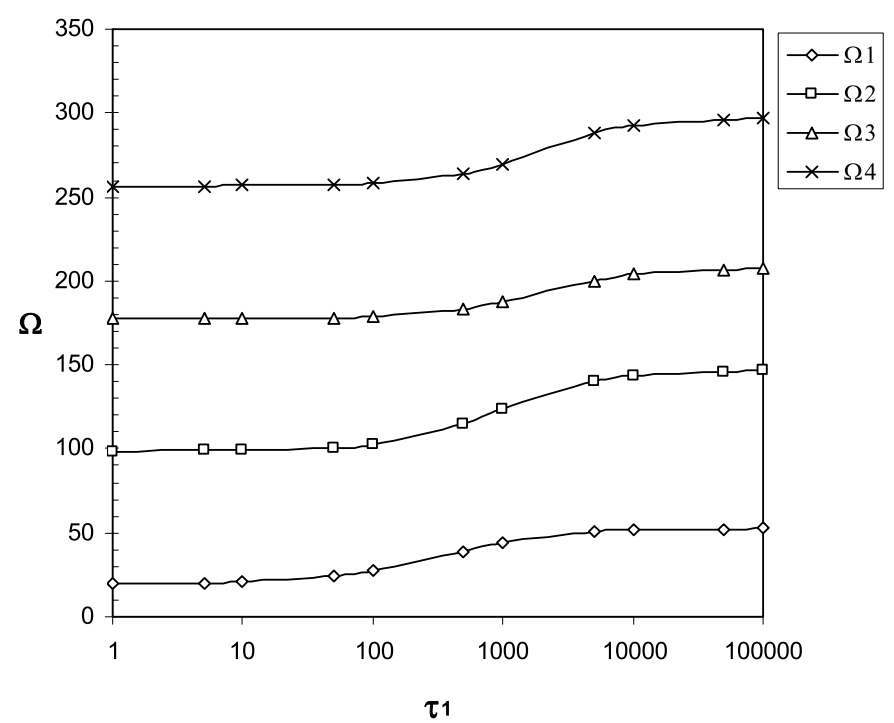

Fig. 4. The first four coupled eigenfrequencies of a SSSS square plate with an elastic point-support $\left(\gamma_{1}=\infty\right)$ at its centre $\left(\xi_{1}=\eta_{1}=0.5\right)$, with respect to the stiffness ratio $\tau_{1}$.

Figures 5-7 give the first two coupled modes of square plates attached with two sprung masses. Three kinds of classical boundary conditions, SSSS, SCSC and SFSF (where SFSF means two opposite edges simply supported and the other two edges are free) are considered. The locations and parameters of the sprung masses are $\xi_{1}=0.4$, $\eta_{1}=0.4, \xi_{2}=0.6, \eta_{2}=0.4, \tau_{1}=\tau_{2}=100.0, \gamma_{1}=\gamma_{2}=0.1$. It is seen from Figs 5-7 that the mode shapes of the coupled system are similar to those of the bare plate although the effect of the sprung masses on the eigenfrequencies is significant. This means that the smaller masses have no significant effects on the mode shapes of the plate. Moreover, it is seen that the effect of the attached masses on the mode shapes of the SFSF plate is larger than that on the SSSS and SCSC plates. For each mode the sprung masses are away from the modal nodes of the bare plate, the larger the effect of the sprung masses on the mode shape and the eigenfrequency of the coupled system. 
Table 5

The first six eigenfrequencies of coupled vibration for a simply supported square plate with two opposite edges rotationally restrained, with one and two sprung masses respectively

\begin{tabular}{|c|c|c|c|c|c|c|}
\hline$K_{r 0}=K_{r 1}$ & $\Omega_{1}$ & $\Omega_{2}$ & $\Omega_{3}$ & $\Omega_{4}$ & $\Omega_{5}$ & $\Omega_{6}$ \\
\hline \multicolumn{7}{|c|}{$\xi_{1}=0.3, \quad \eta_{1}=0.4$} \\
\hline 0.0 & 16.839 & 31.553 & 55.004 & 79.924 & 99.418 & 128.868 \\
\hline 1.0 & 17.121 & 31.803 & 49.970 & 55.259 & 80.345 & 98.900 \\
\hline 10.0 & 18.184 & 32.855 & 52.972 & 56.649 & 82.635 & 99.493 \\
\hline 100.0 & 19.099 & 33.760 & 55.714 & 58.869 & 85.725 & 100.261 \\
\hline 1000.0 & 20.037 & 33.963 & 56.149 & 59.457 & 86.445 & 100.452 \\
\hline$\infty$ & 22.099 & 36.181 & 58.778 & 70.792 & 95.679 & 102.460 \\
\hline \multicolumn{7}{|c|}{$\begin{array}{l}\xi_{1}=0.2, \quad \eta_{1}=0.3, \quad \xi_{2}=0.5, \quad \eta_{2}=0.6, \quad \tau_{1}=\tau_{2}=100.0 \\
\gamma_{1}=\gamma_{2}=0.1\end{array}$} \\
\hline 0.0 & 15.495 & 26.619 & 37.080 & 50.806 & 53.999 & 81.211 \\
\hline 1.0 & 15.804 & 26.651 & 37.138 & 51.321 & 54.534 & 81.645 \\
\hline 10.0 & 17.025 & 26.794 & 37.364 & 53.172 & 57.817 & 83.985 \\
\hline 100.0 & 18.134 & 26.936 & 37.554 & 54.658 & 61.928 & 87.099 \\
\hline 1000.0 & 19.076 & 26.986 & 37.630 & 54.944 & 62.770 & 87.818 \\
\hline$\infty$ & 20.646 & 27.665 & 41.492 & 57.074 & 72.013 & 96.541 \\
\hline
\end{tabular}

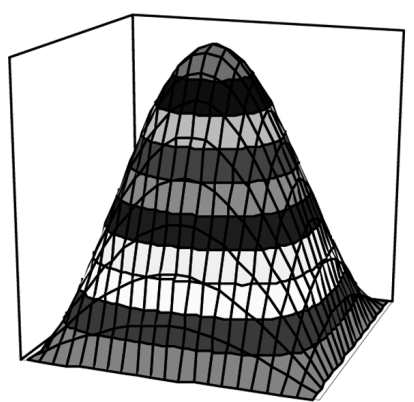

(a)

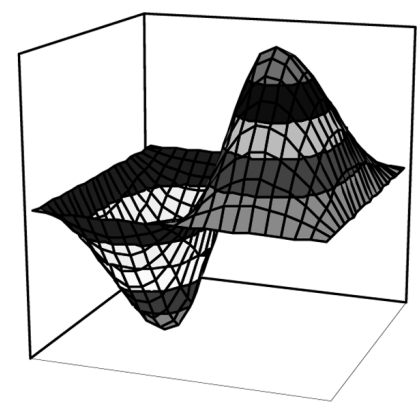

(b)

Fig. 5. The first two coupled modes of a SSSS square plate with two sprung masses $\left(\xi_{1}=0.4, \eta_{1}=0.4, \xi_{2}=0.6, \eta_{2}=0.4\right)$. (a) the mode corresponding to $\Omega_{1}=14.407$, (b) the mode corresponding to $\Omega_{2}=28.267$.

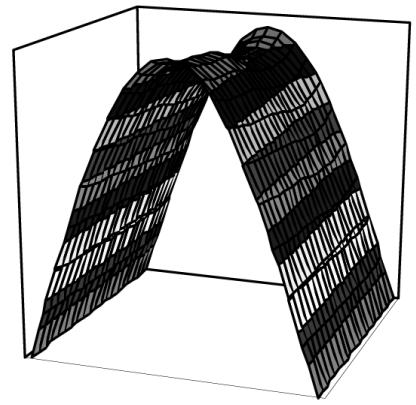

(a)

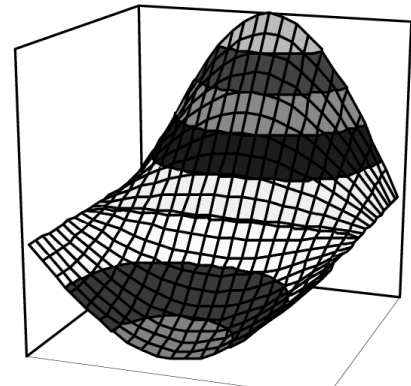

(b)

Fig. 6. The first two coupled modes of a SFSF square plate with two sprung masses $\left(\xi_{1}=0.4, \eta_{1}=0.4, \xi_{2}=0.6, \eta_{2}=0.4\right)$. (a) the mode corresponding to $\Omega_{1}=8.2505$, (b) the mode corresponding to $\Omega_{2}=16.736$. 


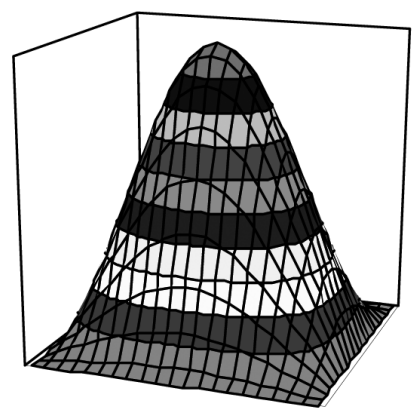

(a)

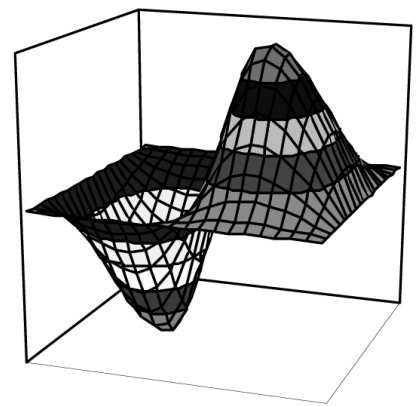

(b)

Fig. 7. The first two coupled modes of a SCSC square plate with two sprung masses $\left(\xi_{1}=0.4, \eta_{1}=0.4, \xi_{2}=0.6, \eta_{2}=0.4\right)$. (a) the mode corresponding to $\Omega_{1}=19.027$, (b) the mode corresponding to $\Omega_{2}=28.609$.

\section{Conclusions}

This paper presents an exact solution of free vibration of a thin rectangular plate attached with sprung masses. The plate is simply supported at two opposite edges and elastically supported at the other edges. The governing differential equations of the free vibration of the plate-spring-mass system are directly solved without introducing further assumptions.

Numerical examples of the solution and parametric studies are provided for comparing the results with published data and for investigating the convergence of the method. As expected, the exact solutions would provide more accurate results than any approximate methods. Thus the solutions provided can be used as benchmark.

A parametric study is conducted to examine the coupled vibration of the sprung mass and plate system.

The solution derived in this paper is general and includes some special cases such as classical boundary conditions, rigid masses, elastic point-supports and rigid vertical supports.

The present analysis is based on a linear model that is a simplification of practical problems. When nonlinear effects are added to the model, suitable approximate calculation should be taken. In that case, the coexistence of solutions and vibration synchronization may play an important role.

\section{Acknowledgements}

The work reported in this paper has been supported by The Leverhulme Trust for the project, Human-Structure Interaction - Applying body biodynamics into structural dynamics.

\section{Appendix}

$$
\begin{gathered}
q_{n i}^{s}(s=1,2,3,4) \text { in Eq. (23) can be written as } \\
\left\{\begin{array}{l}
q_{n i}^{1} \\
q_{n i}^{2} \\
q_{n i}^{3} \\
q_{n i}^{4}
\end{array}\right\}=\left[\begin{array}{llll}
a_{11} & a_{12} & a_{13} & a_{14} \\
a_{21} & a_{22} & a_{23} & a_{24} \\
a_{31} & a_{32} & a_{33} & a_{34} \\
a_{41} & a_{42} & a_{43} & a_{44}
\end{array}\right]^{-1}\left\{\begin{array}{c}
0 \\
b_{1}^{i} \\
0 \\
b_{2}^{i}
\end{array}\right\}
\end{gathered}
$$

in which, when $n<L$

$$
\begin{aligned}
& a_{11}=K_{r 0} \lambda_{n 1}, \quad a_{12}=\lambda_{n 1}^{2}+\frac{\nu(n \pi)^{2}}{\beta^{2}}, \quad a_{13}=K_{r 0} \lambda_{n 2}, \quad a_{14}=-\lambda_{n 2}^{2}+\frac{\nu(n \pi)^{2}}{\beta^{2}}, \\
& a_{21}=K_{r 1} \lambda_{n 1} \cos \lambda_{n 1}-\left(\lambda_{n 1}^{2}+\frac{\nu(n \pi)^{2}}{\beta^{2}}\right) \sin \lambda_{n 1},
\end{aligned}
$$




$$
\begin{aligned}
& a_{22}=-K_{r 1} \lambda_{n 1} \sin \lambda_{n 1}-\left(\lambda_{n 1}^{2}+\frac{\nu(n \pi)^{2}}{\beta^{2}}\right) \cos \lambda_{n 1} \\
& a_{23}=K_{r 1} \lambda_{n 2} \cosh \lambda_{n 2}+\left(\lambda_{n 2}^{2}-\frac{\nu(n \pi)^{2}}{\beta^{2}}\right) \sinh \lambda_{n 2}, \\
& a_{24}=K_{r 1} \lambda_{n 2} \sinh \lambda_{n 2}+\left(\lambda_{n 2}^{2}-\frac{\nu(n \pi)^{2}}{\beta^{2}}\right) \cosh \lambda_{n 2}, \\
& a_{31}=-\lambda_{n 1}^{3}-\frac{(2-\nu)(n \pi)^{2}}{\beta^{2}} \lambda_{n 1}, \quad a_{32}=K_{t 0}, \quad a_{33}=\lambda_{n 2}^{3}-\frac{(2-\nu)(n \pi)^{2}}{\beta^{2}} \lambda_{n 2}, \quad a_{34}=K_{t 0}, \\
& a_{41}=K_{t 1} \sin \lambda_{n 1}+\left(\lambda_{n 1}^{3}+\frac{(2-\nu)(n \pi)^{2}}{\beta^{2}} \lambda_{n 1}\right) \cos \lambda_{n 1} \\
& a_{42}=K_{t 1} \cos \lambda_{n 1}-\left(\lambda_{n 1}^{3}+\frac{(2-\nu)(n \pi)^{2}}{\beta^{2}} \lambda_{n 1}\right) \sin \lambda_{n 1} \\
& a_{43}=K_{t 1} \sinh \lambda_{n 2}-\left(\lambda_{n 2}^{3}-\frac{(2-\nu)(n \pi)^{2}}{\beta^{2}} \lambda_{n 2}\right) \cosh \lambda_{n 2}, \\
& a_{44}=K_{t 1} \cosh \lambda_{n 2}-\left(\lambda_{n 2}^{3}-\frac{(2-\nu)(n \pi)^{2}}{\beta^{2}} \lambda_{n 2}\right) \sinh \lambda_{n 2}, \\
& b_{1}^{i}=\left(\frac{\nu(n \pi)^{2}}{\beta^{2} \lambda_{n 2}}-\lambda_{n 2}\right) \sinh \left(\lambda_{n 2}\left(1-\eta_{i}\right)\right)-\left(\frac{\nu(n \pi)^{2}}{\beta^{2} \lambda_{n 1}}+\lambda_{n 1}\right) \sin \left(\lambda_{n 1}\left(1-\eta_{i}\right)\right)- \\
& K_{r 1}\left[\cosh \left(\lambda_{n 2}\left(1-\eta_{i}\right)\right)-\cos \left(\lambda_{n 1}\left(1-\eta_{i}\right)\right)\right] \\
& b_{2}^{i}=\left(\lambda_{n 2}^{2}-\frac{(2-\nu)(n \pi)^{2}}{\beta^{2}}\right) \cosh \left(\lambda_{n 2}\left(1-\eta_{i}\right)\right)+\left(\lambda_{n 1}^{2}+\frac{(2-\nu)(n \pi)^{2}}{\beta^{2}}\right) \cos \left(\lambda_{n 1}\left(1-\eta_{i}\right)\right)- \\
& K_{t 1}\left[\frac{1}{\lambda_{n 2}} \sinh \left(\lambda_{n 2}\left(1-\eta_{i}\right)\right)-\frac{1}{\lambda_{n 1}} \sin \left(\lambda_{n 1}\left(1-\eta_{i}\right)\right)\right]
\end{aligned}
$$

when $n \geqslant L$

$$
\begin{aligned}
& a_{11}=K_{r 0} \lambda_{n 1}, \quad a_{12}=\frac{\nu(n \pi)^{2}}{\beta^{2}}-\lambda_{n 1}^{2}, \quad a_{13}=K_{r 0} \lambda_{n 2}, \quad a_{14}=\frac{\nu(n \pi)^{2}}{\beta^{2}}-\lambda_{n 2}^{2}, \\
& a_{21}=K_{r 1} \lambda_{n 1} \cosh \lambda_{n 1}+\left(\lambda_{n 1}^{2}-\frac{\nu(n \pi)^{2}}{\beta^{2}}\right) \sinh \lambda_{n 1}, \\
& a_{22}=K_{r 1} \lambda_{n 1} \sinh \lambda_{n 1}+\left(\lambda_{n 1}^{2}-\frac{\nu(n \pi)^{2}}{\beta^{2}}\right) \cosh \lambda_{n 1}, \\
& a_{23}=K_{r 1} \lambda_{n 2} \cosh \lambda_{n 2}+\left(\lambda_{n 2}^{2}-\frac{\nu(n \pi)^{2}}{\beta^{2}}\right) \sinh \lambda_{n 2}, \\
& a_{24}=K_{r 1} \lambda_{n 2} \sinh \lambda_{n 2}+\left(\lambda_{n 2}^{2}-\frac{\nu(n \pi)^{2}}{\beta^{2}}\right) \cosh \lambda_{n 2}, \\
& a_{31}=\lambda_{n 1}^{3}-\frac{(2-\nu)(n \pi)^{2}}{\beta^{2}} \lambda_{n 1}, \quad a_{32}=K_{t 0}, \quad a_{33}=\lambda_{n 2}^{3}-\frac{(2-\nu)(n \pi)^{2}}{\beta^{2}} \lambda_{n 2}, \quad a_{34}=K_{t 0},
\end{aligned}
$$




$$
\begin{aligned}
& a_{41}=K_{t 1} \sinh \lambda_{n 1}-\left(\lambda_{n 1}^{3}-\frac{(2-\nu)(n \pi)^{2}}{\beta^{2}} \lambda_{n 1}\right) \cosh \lambda_{n 1} \\
& a_{42}=K_{t 1} \cosh \lambda_{n 1}-\left(\lambda_{n 1}^{3}-\frac{(2-\nu)(n \pi)^{2}}{\beta^{2}} \lambda_{n 1}\right) \sinh \lambda_{n 1} \\
& a_{43}=K_{t 1} \sinh \lambda_{n 2}-\left(\lambda_{n 2}^{3}-\frac{(2-\nu)(n \pi)^{2}}{\beta^{2}} \lambda_{n 2}\right) \cosh \lambda_{n 2}, \\
& a_{44}=K_{t 1} \cosh \lambda_{n 2}-\left(\lambda_{n 2}^{3}-\frac{(2-\nu)(n \pi)^{2}}{\beta^{2}} \lambda_{n 2}\right) \sinh \lambda_{n 2}, \\
& b_{1}^{i}=\left(\lambda_{n 1}-\frac{\nu(n \pi)^{2}}{\beta^{2} \lambda_{n 1}}\right) \sinh \left(\lambda_{n 1}\left(1-\eta_{i}\right)\right)-\left(\lambda_{n 2}-\frac{\nu(n \pi)^{2}}{\beta^{2} \lambda_{n 2}}\right) \sinh \left(\lambda_{n 2}\left(1-\eta_{i}\right)\right)- \\
& K_{r 1}\left[\cosh \left(\lambda_{n 2}\left(1-\eta_{i}\right)\right)-\cosh \left(\lambda_{n 1}\left(1-\eta_{i}\right)\right)\right] \\
& b_{2}^{i}=\left(\lambda_{n 2}^{2}-\frac{(2-\nu)(n \pi)^{2}}{\beta^{2}}\right) \cosh \left(\lambda_{n 2}\left(1-\eta_{i}\right)\right)-\left(\lambda_{n 1}^{2}-\frac{(2-\nu)(n \pi)^{2}}{\beta^{2}}\right) \cosh \left(\lambda_{n 1}\left(1-\eta_{i}\right)\right)- \\
& K_{t 1}\left[\frac{1}{\lambda_{n 2}} \sinh \left(\lambda_{n 2}\left(1-\eta_{i}\right)\right)-\frac{1}{\lambda_{n 1}} \sinh \left(\lambda_{n 1}\left(1-\eta_{i}\right)\right)\right]
\end{aligned}
$$

\section{References}

[1] B.R. Ellis and T. Ji, Human-structure interaction in vertical vibrations, Structures and Buildings, the Proceedings of Civil Engineers 122(1) (1997), 1-9.

[2] S.C. Fan and Y.K. Cheung, Flexural free vibrations of rectangular plates with complex support conditions, Journal of Sound and Vibration 93(1) (1984), 81-94.

[3] C.S. Kim and S.M. Dickinson, The flexural vibration of rectangular plates with point supports, Journal of Sound and Vibration 117(2) (1987), 249-261.

[4] D. Zhou, Vibrations of point-supported rectangular plates with variable thickness using a set of static tapered beam functions, International Journal of Mechanical Sciences 44(1) (2002), 149-164.

[5] Y.K. Cheung and D. Zhou, The free vibrations of rectangular composite plates with point-supports using static beam functions, Composite Structures 44(2-3) (1999), 145-154.

[6] D. Zhou, Y.K. Cheung and J. Kong, Free vibration of thick, layered rectangular plates with point supports by finite layer method, International Journal of Solids and Structures 37(10) (2000), 1483-1499.

[7] L.A. Bergman, J.K. Hall, G.G.G. Lueschen and D.M. McFarland, Dynamic Green's functions for Levy plates, Journal of Sound and Vibration 162(2) (1993), 281-310.

[8] Q.S. Li, An exact approach for free vibration analysis of rectangular plates with line-concentrated mass and elastic line-support, International Journal of Mechanical Sciences 45(4) (2003), 669-685.

[9] D.R. Avalos, H.A. Larrondo and P.A.A. Laura, Transverse vibrations of a circular plate carrying an elastically mounted mass, Journal of Sound and Vibration 177(2) (1993), 251-258.

[10] D.R. Avalos, H. Larrondo and P.A.A. Laura, Vibrations of a simply supported plate carrying an elastically mounted concentrated mass, Ocean Engineering 20(2) (1993), 195-200.

[11] W.O. Wong, The effect of distributed mass loading on plate vibration behavior, Journal of Sound and Vibration 252(3) (2002), 577-583.

[12] J.S. Wu, H.M. Chou and D.W. Chen, Free vibration analysis of a rectangular plate carrying multiple various concentrated elements, Proceedings of the Institution of Mechanical Engineers-Part K: Journal of Multi-body Dynamics 217(2) (2003), 171-182.

[13] R.G. Jacquot, Suppression of random vibration in plates using vibration absorbers, Journal of Sound and Vibration 248(4) (2001), 585-596.

[14] M. Chiba and T. Sugimoto, Vibration characteristics of a cantilever plate with attached spring-mass system, Journal of Sound and Vibration 260(2) (2003), 237-263.

[15] K.M. Liew, S. Kitipornchai and M.K. Lim, Vibration of Mindlin plates on point supports using constraint functions, ASCE Journal of Engineering Mechanics 120(3) (1994), 499-513.

[16] A.J. McMillan and A.J. Keane, Shifting resonances from a frequency band by applying concentrated masses to a thin rectangular plate, Journal of Sound and Vibration 192(2) (1996), 549-562.

[17] A.J. McMillan and A.J. Keane, Vibration isolation in a thin rectangular plate using a large number of optimally positioned point masses, Journal of Sound and Vibration 202(2) (1997), 219-234.

[18] S. Adhikari, M.I. Friswell, K. Lonkar and A. Sarkar, Experimental case studies for uncertainty quantification in structural dynamics, Probabilistic Engineering Mechanics 24(4) (2009), 473-492. 
[19] S. Adhikari and A. Srikantha Phani, Random eigenvalue problems in structural dynamics: experimental investigations, AIAA Journal 48(6) (2010), 1085-1097.

[20] D. Zhou and T. Ji, Free vibration of rectangular plates with internal column supports, Journal of Sound and Vibration 297(1-2) (2006), $146-166$.

[21] D.J. Gorman, Free Vibration Analysis of Rectangular Plates, Oxford, NY, Elsevier, 1982. 

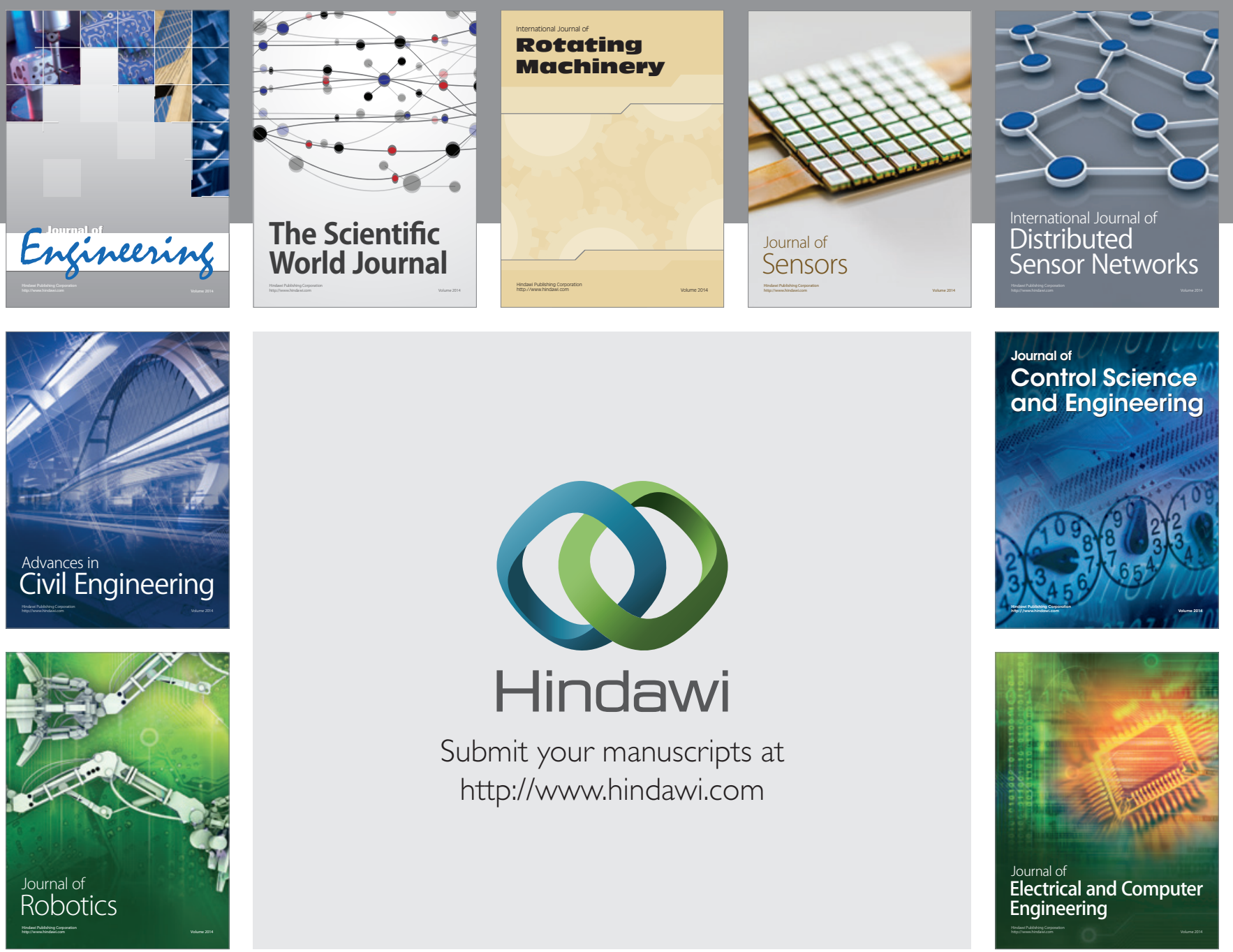

Submit your manuscripts at

http://www.hindawi.com
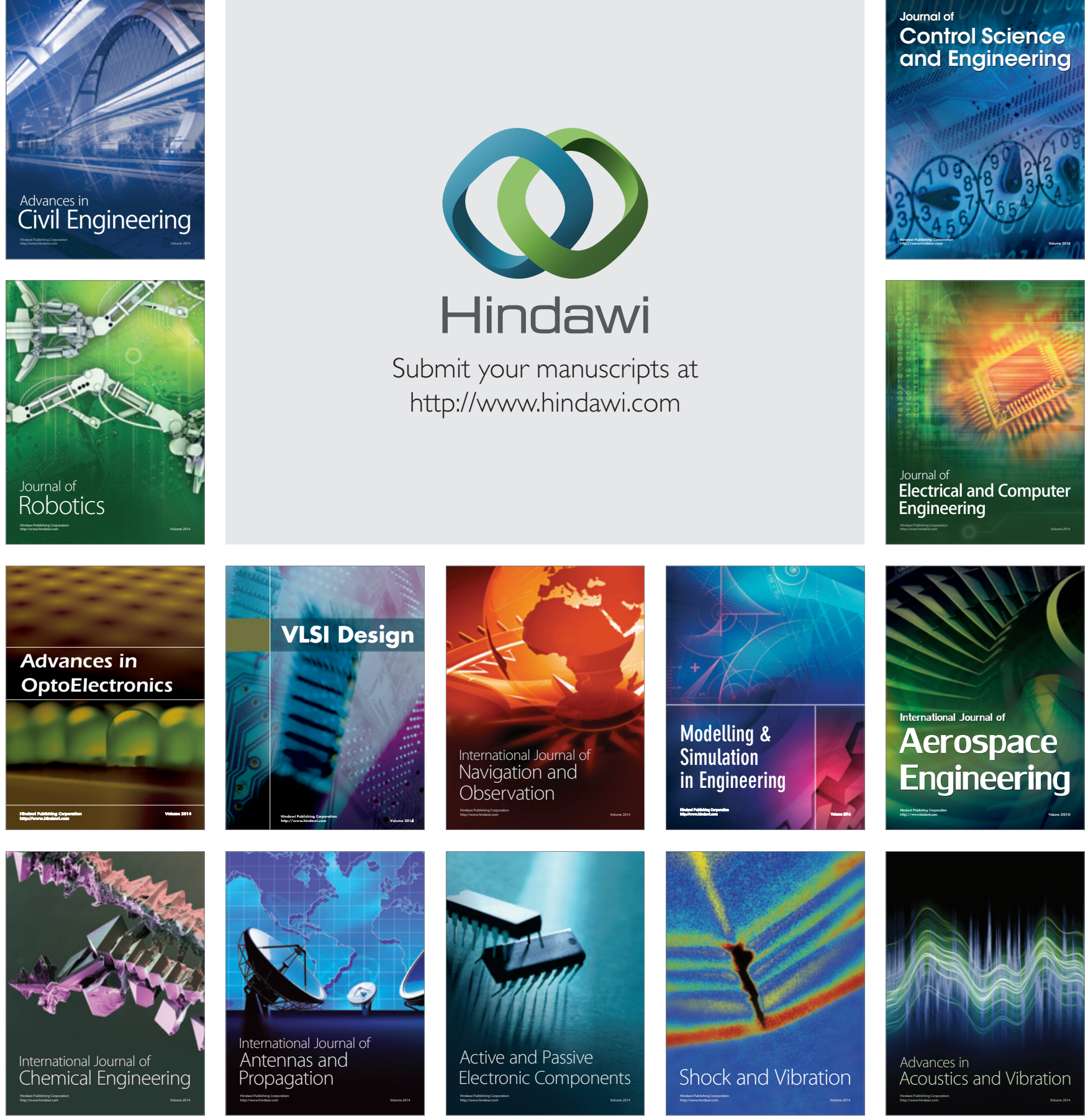